\title{
Calibration of The Spatial Pose Between Inertial and Visual Sensors With An Inclinometer
}

\author{
Shubin Zheng ${ }^{*}$, Xiaodong Chai, Xinchang Liu, Huaiqing Lin \\ School of Urban Railway Transportation, Shanghai University of Engineering Science, Shanghai 201620, China
}

\begin{abstract}
An improved inertial and visual sensors calibration method is proposed in this paper, which is the method of fractional step to calibrate between inertial and visual sensors. The relationship of the rotation between inertial and vision sensors can be obtain by solving the improvement of hand-eye calibration equations. The inclinometer sensor is introduced in the process of calibration and the output of inclinometer data is utilized to optimize the rotation matrix between inertial and vision sensors; the translation between inertial and vision sensors can be obtain through solving the basic hand-eye calibration equations. The experiment results demonstrate that the method can precisely calibrate the relative position of the space between inertial and visual sensors.
\end{abstract}

Keywords: Calibration, hand-eye, inertial sensor, visual sensor, relative position, rotation matrix.

\section{INTRODUCTION}

The track is the basis of train running, it is directly influence the safety and comfort running of train $[1,2]$, Adjustment and control of the track should adopt the static measurement parameters which established in the geodetic coordinates according to the national railway maintenance standards [3]. Binding the inertial measurement unit and a vision sensor (IMU-Camera) in a motion platform, which can quickly and accurately obtain static geometric parameters in earth coordinates of the track through analyzing both signal integration. This process needs to calibrate the IMUcamera's relative position, but the fusion accuracy will decrease because of calibration error. Therefore, the accurate calibration relative position of the inertial and vision sensors has a great significance on getting static geometric parameters of the track.

At present, both domestic and foreign have widely studied on IMU-Camera calibration methods, which mainly divided into the robot hand- eye calibration method and filtering estimation method. Calibrating the spatial position between inertial and vision sensors by improving the robot hand-eye calibration methods [4], the manual operation is difficult and hard to ensure accuracy in this method. Using the method of filtering estimation to calibrate inertial and visual sensors relative position [5], which cannot be accurately calibrated in the condition of the larger initial errors or interfered by the larger system noise. Regarding the calibration board as an intermediary and obtaining the relative position between visual sensor and the world coordinate system, the IMU and the world coordinate system through the calibration $[6,7]$. Finally, the calibration get the spatial relative position between inertial and visual sensors, but the ancillary equipment is so expensive in general that this method is difficult to achieve.

This paper obtained rotation relationship of the inertial measurement unit relative visual sensor through improving hand-eye calibration equations. In order to improve the calibration precision, the process of calibration only used the pitch and the roll angle of the inertial measurement unit. Using the output of inclinometer sensor information, the rotation obtained between the inertial and vision sensors had been solved and optimized. Translation between inertial and vision sensors was obtained by solving hand-eye calibration method.

\section{CALIBRATION BETWEEN INERTIAL AND VI- SION SENSORS}

Calibration between inertial and vision sensors is to solve the relationship of rotation and translation between inertial and visual sensor. The calibration system consists of inertial measurement unit, visual sensor and calibration board, spatial relative position as shown in Fig. (1), the establishment of coordinates are as follows.

(1) The word coordinates $\{w\}$, the coordinate origin is located in the lower-left corner of calibration board, the axis $\mathrm{x}$ is parallel to an edge of the calibration board, the axis $\mathrm{y}$ is perpendicular to the direction of calibration board, the axis $\mathrm{z}$ is perpendicular to another edge of the calibration board in a parallel direction and the perpendicular direction is upward. $\{\mathrm{w}\}$ determines the absolute position of inertial and visual sensors in the world coordinate.

(2) Visual sensor coordinate system $\{c\}$, the origin of coordinates is located in optical center of visual sensor, the axis $\mathrm{x}$ and $\mathrm{y}$ are parallel to the plane of vertical axis the horizontal axis of the image. The axis $z$ parallel to the optical axis of the sensor. 


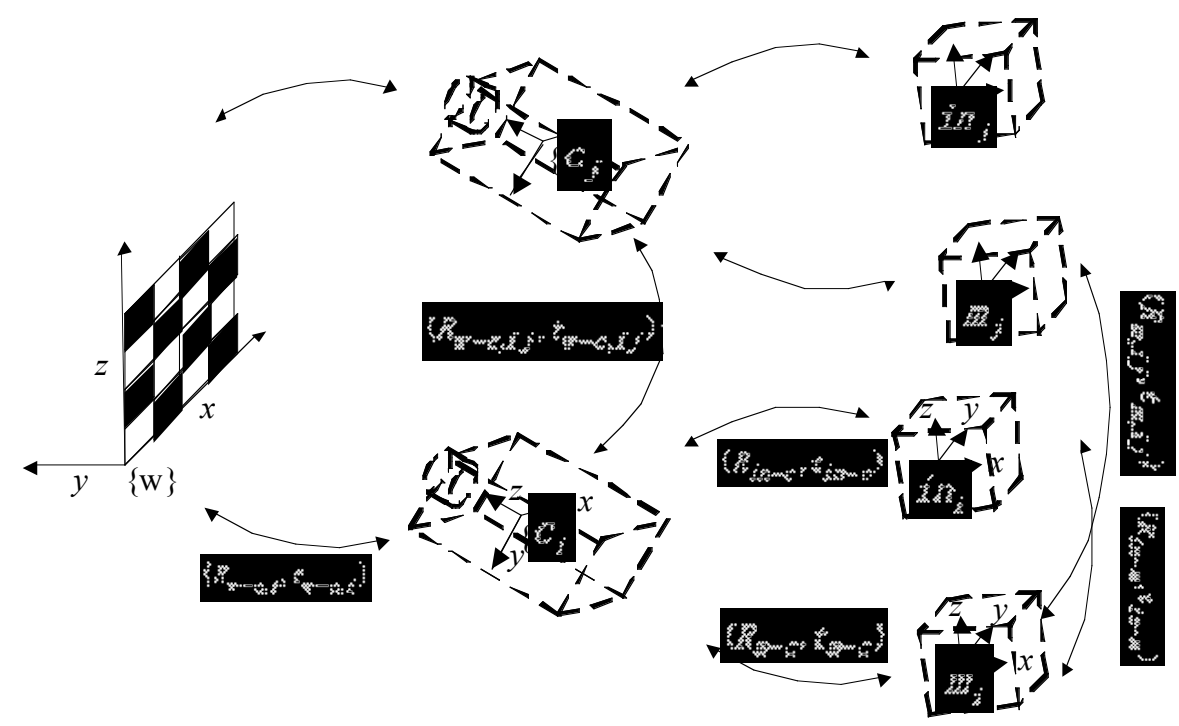

Fig. (1). The coordinate relative positions of mobile platform before and after IMU coordinates, camera coordinates, word coordinates.

(3) Inertial measurement unit coordinates $\{\mathrm{m}\}$, the origin of coordinates is located in the center of gravity of the inertial measurement unit. The axis $\mathrm{x}$ and $\mathrm{y}$ are parallel to the long axis and short axis of the inertial measurement unit, the axis $\mathrm{z}$ is obtained by the right-hand rule. $\{\mathrm{m}\}$ is used to measure the object linear acceleration and rotating angular velocity;

(4) Inclinometer coordinates $\{$ in $\}$, the axis $x$ and $y$ are parallel respectively to the long axis and short axis of the inertial measurement unit, the output of $\{$ in $\}$ is the angle of the axis $\mathrm{x}$ and $\mathrm{y}$ around the rotation.

(5) Inertial sensor Euler angle coordinate system is $\{\mathrm{e}\}$, which defines time coordinate system of inertial sensor.

\subsection{Solving the Rotating Relationship between Inertial and Visual Sensors}

The system consists of an inertial measurement unit, inclinometer and camera. First of all, using the output of inertial sensor the angular velocity and accelerometer data to solve three axis Euler angle of the inertial sensor, at the same time, While using the camera calibration to calculate the spatial transformation relationship between the camera and the world coordinate system, and then obtaining the space transform relation between the camera and the inertial sensors. In this system, the stability of inclinometer output of the Euler angle slightly is higher than using the inertial sensors' output of acceleration data and angular velocity data calculated by three axis Euler angle. So, using the output information of inclinometer can improve the estimation accuracy of the rotation relationship between inertial and vision sensors.

\subsubsection{Three Axis Euler Angle Estimation Algorithm}

The direction quaternion of inertial sensor is represented as $q=\left[q_{1}, q_{2}, q_{3}, q_{4}\right]$, the sensor measurement angular velocity is represented as $\left(\omega_{x}, \omega_{y}, \omega_{z}\right)$,

$$
\text { Definition } S_{\omega}=\left[0, \omega_{x}, \omega_{y}, \omega_{z}\right]
$$

Therefore, the first-order differential of direction quaternion $q_{\omega, t}$

$$
\dot{q}_{\omega, t}=\frac{1}{2} q_{\omega, t} \otimes S_{\omega}
$$

" $\otimes "$ is the quaternion multiplication operator. Estimate inertial sensor direction quaternion by formula (1) as follow:

$$
\min _{q} f(q, a)=q^{*} \otimes g \otimes q-a
$$

Among them

$$
a=\left[0, a_{x}, a_{y}, a_{z}\right] q^{*}=\left[q_{1},-q_{2},-q_{3},-q_{4}\right]
$$

and $g=[0,0,0,1]$, from (2), we have

$f(q, a)=\left[\begin{array}{c}2\left(q_{2} q_{4}-q_{1} q_{3}\right)-a_{x} \\ 2\left(q_{1} q_{2}+q_{3} q_{4}\right)-a_{y} \\ 2\left(0.5-q_{2}^{2}-q_{3}^{2}\right)-a_{z}\end{array}\right]$

The appropriate Jacobian matrix is:

$$
J(q)=\left[\begin{array}{cccc}
-2 q_{3} & 2 q_{4} & -2 q_{1} & 2 q_{2} \\
2 q_{2} & 2 q_{1} & 2 q_{4} & 2 q_{3} \\
0 & -4 q_{2} & -4 q_{3} & 0
\end{array}\right]
$$

Define $q_{\text {est }, t}$ as estimated the inertial sensor direction quaternion at the moment of $t$ and $q_{e s t, t}$ as the first-order differential of $q_{e s t, t}$, then from formula (3) and (4), the error direction vector of $\dot{q}_{\text {est }, t}$ defines as $\dot{q}_{\text {err }, t}$ :

$$
\dot{q}_{\text {err }, t}=\frac{J\left(q_{e s t, t-1}\right)^{T} f\left(q_{e s t, t-1}, a_{t}\right)}{\left\|J\left(q_{e s t, t-1}\right)^{T} f\left(q_{e s t, t-1}, a_{t}\right)\right\|}
$$

Then, we have 
$\dot{q}_{\text {est }, t}=\dot{q}_{\omega, t}-\beta \dot{q}_{\text {err }, t}$

$q_{e s t, t}=q_{e s t, t-1}+\dot{q}_{\text {est }, t} \Delta t$

$\beta$ is the divergence rate parameters, the Euler angle of the inertial sensor calculated by the following formula:

Pitch $=-a \sin \left(2\left(q_{2} q_{4}+q_{1} q_{3}\right)\right)$

Roll $=a \tan \left(2\left(q_{3} q_{4}-q_{1} q_{2}\right) / 2 q_{1}^{2}-1+2 q_{4}^{2}\right)$

$Y a w=a \tan \left(2\left(q_{2} q_{3}-q_{1} q_{4}\right) / 2 q_{1}^{2}-1+2 q_{2}^{2}\right)$

2.1.2. Calculation of the Spatial Transformation Between the Camera and the Inertial Sensors

Using visual sensor to take $m$ image of the checkerboard calibration plate in the scene from different perspective and define each image as $I_{i}$. Recording the inertial sensor of the Roll angle $\alpha_{i}$, the Pitch angle $\beta_{i}$ and the Yaw angle $\gamma$ for each $I_{i}$ at the moment. At the same time, using camera calibration $[8,9]$ to obtain the rotation matrix $R_{w-c, i}$ of the world coordinate system to the camera coordinate system at the moment.

The related data of any of arbitrary $I_{i}$ image and $I_{j}$ image can be obtained according to the basic hand -eye calibration equation $\mathrm{c}$ :

$R_{w-c, i j} * R_{m-c}=R_{m-c} * R_{e-m, i j}$

Among them, $R_{w-c, i j}=R_{w-c, i} * R_{w-c, \mathrm{j}}^{-1}$ represent the rotation matrix of camera coordinate system at the moment $i, j, R_{\mathrm{e}-\mathrm{m}, i j}=R_{\mathrm{e}-\mathrm{m}, i} * R_{e-m, \mathrm{j}}^{-1}$ represent the rotation matrix of inertial sensor coordinate system at the moment $i, j, R_{\mathrm{e}-\mathrm{m}, i}$ represent the rotation matrix of inertial sensor from Euler angle coordinates to inertial sensor coordinate system at the moment $i$.

$R_{e-m}=\left(\begin{array}{ccc}1 & 0 & 0 \\ 0 & \cos \alpha & \sin \alpha \\ 0 & -\sin \alpha & \cos \alpha\end{array}\right) \times\left(\begin{array}{ccc}\cos \beta & 0 & -\sin \beta \\ 0 & 1 & 0 \\ \sin \beta & 0 & \cos \beta\end{array}\right) \times\left(\begin{array}{ccc}\cos \gamma & \sin \gamma & 0 \\ -\sin \gamma & \cos \gamma & 0 \\ 0 & 0 & 1\end{array}\right)(12)$

Association equation (11) with (12) can obtain:

$R_{w-c, i j} \times R_{m-c} \times\left(\begin{array}{c}-\sin \beta_{j} \\ \sin \alpha_{i} \cos \beta_{j} \\ \cos \alpha_{i} \cos \beta_{j}\end{array}\right)=R_{m-c} \times\left(\begin{array}{c}-\sin \beta_{i} \\ \sin \alpha_{i} \cos \beta_{i} \\ \cos \alpha_{i} \cos \beta_{i}\end{array}\right)$

Get substitution of $R_{w-c, i j}$ and the Euler angle $\alpha_{i} ! \beta_{i} \alpha_{j} \beta_{j}$ of inertial sensor into equation (13) and denote the vector representation of the rotation matrix $R_{m-c}$ as $V_{m-c}$, We can get the following linear equation :
$A_{i, j} V_{m-c}=0$

Where $A_{i, j}$ is the coefficient matrix in equation (13) after simplification.

Any combination of two $m$ images can obtain $C_{m}^{2}$ kinds of different image pairs. These image pairs use the above calculation method, which can obtain $C_{m}^{2}$ kinds of equations from the inertial sensor coordinate system to the camera coordinate system rotation matrix $R_{m-c}$ that the form as type (14), i.e.

$A V_{m-c}=\left(\begin{array}{c}A_{1,2} \\ A_{1,3} \\ \vdots \\ A_{m-1, m}\end{array}\right) V_{m-c}=0$

$R_{m-c}$ is a rotation matrix and $R_{m-c}^{T} R_{m-c}=E_{3}$, therefore, to solve the rotation matrix $R_{m-c}$ can be transformed into a constrained nonlinear optimization as the following:

$\arg _{V_{m-c}} \min V_{m-c}^{T} A^{T} A V_{m-c}$

s.t. $R_{m-c}^{T} R_{m-c}=E_{3}$

The rotation matrix $R_{m-c}$ between the inertial sensor coordinate system and the camera coordinate system can be obtained by solving equations above.

\subsubsection{The Calibration Method of Rotation Relationship between the Inertial Measurement Unit and Inclinometer}

The inclinometer and inertial measurement unit is fixed on the same plane in this system, so it can be directly used Euler angle value via the output of inclinometer as well as rotation matrix calibration method based on Roll angle and Pitch angle in section 2.1.2, calculating rotation matrix $R_{i n-c}$ between the inclinometer coordinate and camera coordinate system with the rotation matrix $R_{i n-m}$ between inclinometer coordinate system and inertial measurement unit coordinate system is:

$R_{i n-m}=R_{m-c}^{-1} R_{i n-c}$

\subsubsection{Optimization of the Rotation Relationship between the Camera and the Inertial Measurement Unit}

Transforming output of two axis Euler angle of the inclinometer to the inertial sensor Euler angle coordinate system, associating with the Euler angle obtained by the inertial sensors angle integral algorithm weighted fusion through the below equation:

$\left[\begin{array}{l}\hat{\alpha}_{1} \\ \hat{\beta}_{1} \\ \hat{\gamma}_{1}\end{array}\right]=\lambda R_{i n-m}^{t-1}\left[\begin{array}{l}x_{i} \\ y_{i} \\ \gamma_{i}\end{array}\right]+(1-\lambda)\left[\begin{array}{c}\alpha_{i} \\ \beta_{i} \\ \gamma_{i}\end{array}\right]$ 
$\lambda$ is the modulation parameters, $R_{i n-m}^{t-1}$ is the estimated rotation matrix at the former moment. Using the information above, creating the objective function as the equation (15) and solving the rotation matrix $R_{m-c}^{t}$ between the inertia and visual sensors at the current moment.

\subsection{Solution of the Translation Relations between Inertia and Vision Sensors}

According to the basic hand-eye calibration equation, we have:

$\left[\begin{array}{cc}R_{w-c, i j} & t_{w-c, i j} \\ 0 & 1\end{array}\right]\left[\begin{array}{cc}R_{m-c} & t_{m-c} \\ 0 & 1\end{array}\right]=\left[\begin{array}{cc}R_{m-c} & t_{m-c} \\ 0 & 1\end{array}\right]\left[\begin{array}{cc}R_{m, i j} & t_{m, i j} \\ 0 & 1\end{array}\right]$

In the equation, $t_{w-c, i j}$ is the translation vector for camera coordinate system between the two times $i, j, R_{m, i j}=R_{e-m, i} * R_{e-m, j}^{-1}$ is the rotation matrix for the inertial sensor coordinate system between the two times $i, j$, $t_{m, i j}$ is the translation vector for the inertial sensor in $i, j$, which is obtained by the quadratic integral through the output of inertial sensor acceleration information.

According to the equation (20) can obtain:

$$
\left(R_{w-c, i j}-E_{3}\right) t_{m-c}=R_{m-c} t_{m, i j}-t_{w-c, i j}
$$

As the rank of $R_{w-c, i j}-E_{3}$ is 2 , equation (21) can only provide two independent constraint equations about $t_{m-c}$. Therefore, we need to control the movement platform for at least two motion, to get two (or more) constraint equations like equation (21), and then get the constraint equations as follows:

$$
C t_{m-c}=D
$$

where $C=\left[\begin{array}{l}R_{w-c, i j}^{-1}-E_{3} \\ R_{w-c, i j}^{2}-E_{3}\end{array}\right], D=\left[\begin{array}{l}R_{w-c, i j}^{-1} t_{m-c}^{1}-t_{w-c, i j}^{1} \\ R_{m-c, i j}^{2} t_{m-c}^{2}-t_{w-c, i j}^{2}\end{array}\right]$, then:

$t_{m-c}=C^{+}+D$

where $C^{+}$is the pseudo-inverse of matrix $C$, then get $t_{m-c}$.

\section{EXPERIMENTAL ANALYSIS}

Calibration test bench as shown in Fig. (2), industrial camera uses AVT Stingray MV-F125C, inertial measurement unit uses IMU-F100A5 manufactured by German GeneSys company, inclinometer uses LCF2000. Calibration platform is a motion platform of six freedom degrees. After setting the parameters of the movement control cards, which can control card library function to the operate the motion platform accordingly.

Inertial coordinate system which is obtained by the calibration method of visual sensor coordinate system is relative to the axis of the change of the Euler angle curve as shown in Fig. (3). As shown in the figure, with the increase of test

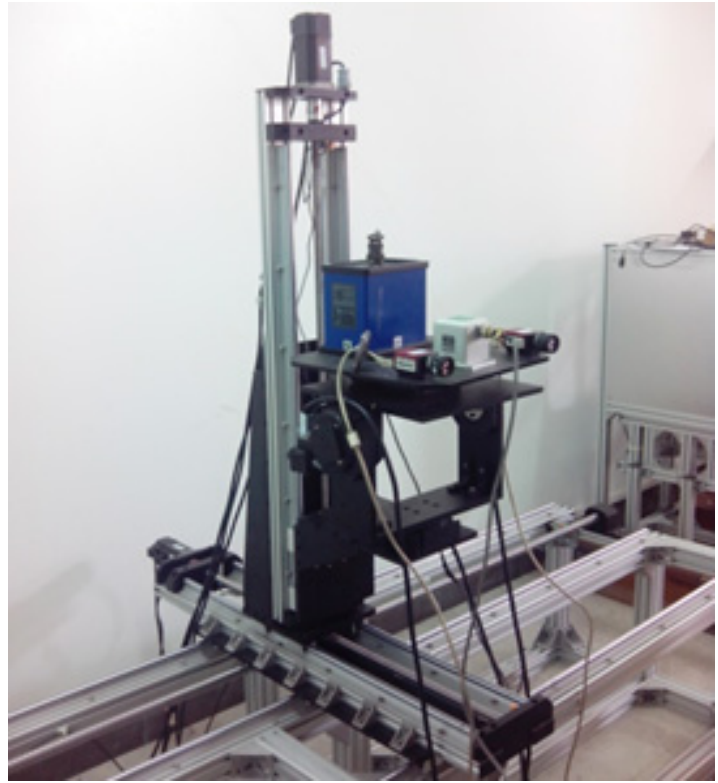

Fig. (2). IMU-camera calibration test bench.
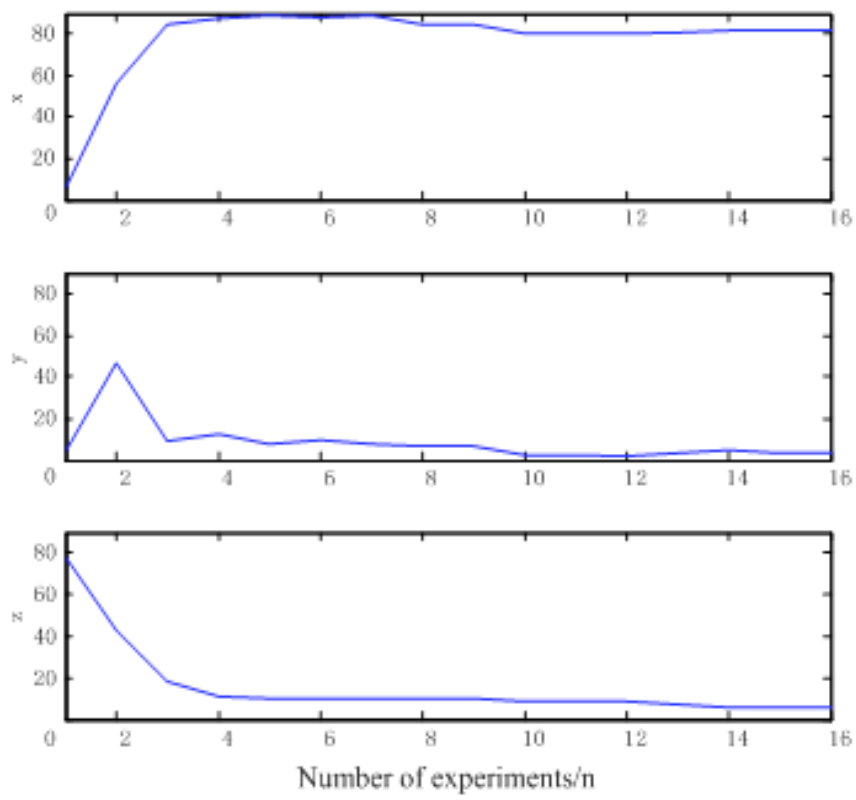

Fig. (3). Around the $x, y, z$ three axis euler angle measurements.

times, the measured results gradually stabilized. Measurement value of space relative position between inertia and visual sensors is $[3.5,-8.2,-5.8]$.

In order to verify the the method accuracy proposed in this paper, space relative position of inertia and visual sensors is accurately calibrated by using high precision $3 \mathrm{D}$ laser scanner and calibration. Compared with the calibration results and calibration results obtained in this paper, when the experimental data is greater than 15 groups, the maximum error of three axis Euler angle is less than 1 degree, the maximum error of space relative position is less than $0.8 \mathrm{~cm}$.

\section{CONCLUSION}

This paper uses two-step method for inertia and visual sensors relative position calibration. Through the improved 
hand-eye calibration equation can solve the rotating relationship between inertia and visual sensors. At the same time, introducing the inclinometer sensor for optimized solution and making the calibration precision greatly improve. On this basis, the accuracy of translation quantity between sensors which is obtained by basic hand-eye calibration equation must be improved. The experimental results show that this method can precisely calibrate the relative position between inertia and visual sensors.

\section{CONFLICT OF INTEREST}

The authors confirm that this article content has no conflict of interest.

\section{ACKNOWLEDGEMENTS}

This work is supported by Shanghai Municipal Natural Science Foundation (12ZR1412300), Key Technology R\&D Project of Shanghai Committee of Science and Technology (13510501300), Innovation Program of Shanghai Municipal Education Commission Scientific (12ZZ184), and Discipline Construction Project for Transportation Engineering (13SC002).

\section{REFERENCES}

[1] Y. Cai, Y. Wang, and Z. Cao, "Trains run time caused by vibration of foundation research track irregularity," Rock and Soil Mechanics, vol. 33, no. 2, pp. 327-335, 2012.

[2] G. Zhang, J. Li, and Z. Yang, "The study on low-speed maglev rail irregularity power spectrum," Journal of the China Railway Society, vol. 33, no. 10, pp. 73-78, 2011.

[3] G. Zhao, "High-speed rail track geometry status detection and track fine adjustment," Beijing: The Beijing-shanghai high-speed railway co., LTD, 2010.

[4] J. Lobo, and J. Dias, "Relative pose calibration between visual and inertial sensors," International Journal of Robotics Research vol. 26, no. 06, pp. 561-575, 2007.

[5] F. M. Mirzaei, and S. I. Roumeliotis, "A Kalman filter based algorithm for IMU-camera calibration: Observability analysis and performance evaluation," IEEE Transactions on Robotics, vol. 24, no. 05, pp. 1143-1156, 2008.

[6] A. E. Johnson, R. Willson, J. Goguen, J. Alexander, and D. Meller, "Field testing of the Mars exploration rovers descent image motion estimation system," IEEE International Conference on Robtics and Automation, Barcelona, Spain, pp. 4463-4469, 2005.

[7] A. E. Johnson, J. F. Montgomery, and L. H. Matthies, "Vision guided landing of an autonomous helicopter in hazardous terrain," IEEE International Conference on Robtics and Automation, Barcelona, Spain, pp. 3966-3971, 2005.

[8] B. K. P. Horn, "Closed-form solution of solution of absolute orientation using unit quaternions," Journal of the Optical Society of America, vol. 4, no. 4, pp. 629-642, 1987.

[9] S. Ma, and Z. Zhang, "Computer vision - Algorithms and theory of computation basis," Beijing: Science Press 2003. 